\title{
A HIGH RESOLUTION WIRE SCANNER BEAM PROFILE MONITOR WITH A MICROPROCESSOR DATA ACQUISITION SYSTEM*
}

\section{R.I. Cutler, D.L. Mohr, J.K. Whittaker, and N.R. Yodert National Bureau of Standards} Washington, DC 20234

\section{Abstract}

A beam profile monitor has been constructed for the NBS-LANL Racetrack Microtron. The monitor consists of two perpendicular $30 \mu \mathrm{m}$ diameter carbon wires that are driven through an electron beam by a pneumatic actuator. A long-lifetime, electroformed nickel bellows is used for the linear-motion vacuum feedthrough. Secondary emission current from the wires and a signal from a transducer measuring the position of the wires are simultaneously digitized by a microprocessor to yield beam current density profiles in two dimensions. The wire scanner is designed for use with both pulsed and $\mathrm{cw}$ beams.

\section{Introduction}

The NBS-LANL Racetrack Microtron (RTM) will produce a $185 \mathrm{MeV}$ electron beam ranging in diameter from $1 \mathrm{~mm}$ to $3 \mathrm{~mm}$, with currents of $0-500 \mu \mathrm{A}$, either $\mathrm{CW}$ (100\% duty factor), or pulsed with repetition rates of $0.1-100 \mathrm{kHz}$. In order to steer such beams through the RTM, a high resolution, non-destructive, beam-profile monitoring system is being developed. This system will consist of forty wire scanners, each of which can intercept the beam with a fine carbon wire to measure beam position and current distributions. A microprocessor-based wire-scanner control and data accumulation system will be used. The beam profile monitoring system will present the accelerator operator a real-time, simultaneous display of the beam size and position at 2 of the 40 scanner positions, allowing the effects of focussing and steering to be observed. The location of the wire scanners in the RTM is shown in figure 1.

\section{Wire Scanners}

The wire scanner shown in figure 2 consists of an actuator that moves two orthogonal wires through the electron beam. The secondary emission current produced in the wires by the impinging beam is measured as a function of wire position. This yields data on beam size, position, and current density distribution.

The wires are $30 \mu \mathrm{m}$ diameter carbon filaments mounted on insulated standoffs on a metal frame. The two wires are mounted in an "L" pattern and connected electrically. The wires are driven through the beam at a $45^{\circ}$ angle to the wires so that in a single sweep through the beam, the two wires measure the beam current distribution projections on two orthogonal axes. Current from both wires is transmitted through a high vacuum electrical feedthrough to a low-noise, flexible, co-axial cable to a high-gain, DC-coupled amplifier.

Motive power for the wire scanner is provided by a pneumatic actuator with an electroformed nickel bellows as the vacuum seal. Both the bellows and pneumatic actuator have been tested for lifetimes in excess of $2 \times 10^{7}$ cycles operating at $8-10$ cycles per second. Horizontal and vertical beam position and current distributions measured at this rate are fast enough for

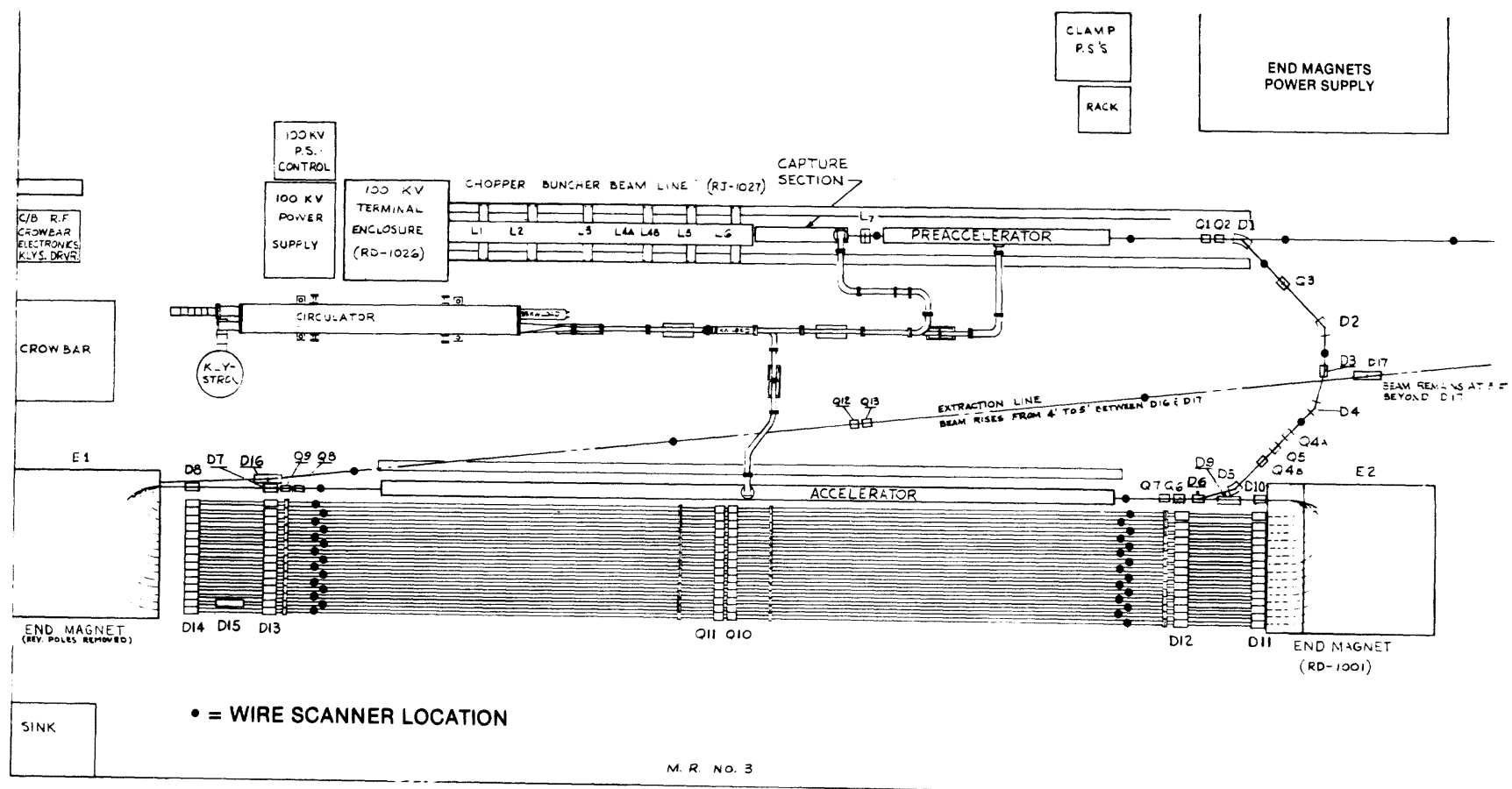

Figure 1. Wire scanner locations in NBS-LANL RTM

*Work supported in part by the Division of Nuclear Physics, U.S. Department of Energy.

tOn IPA agreement from the University of Maryland.

U.S. Government work not protected by U.S. copyright. 


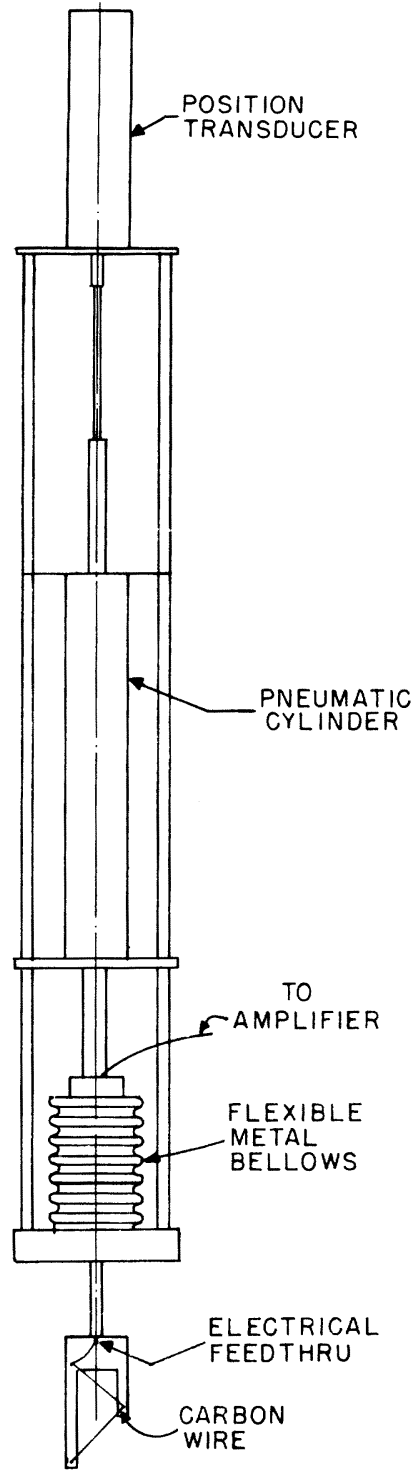

Figure 2. Sketch of wire scanner.

real-time human interaction with the RTM controls with no perceptible delay. Cycling of the actuator is controlled by a commercially available pneumatic logic system which is adjustable over a wide range of cycle times. At 8 sweeps per second each cycle consists of $50 \mathrm{~ms}$ to extend the actuator $5 \mathrm{~cm}$, a $75 \mathrm{~ms}$ wait, $50 \mathrm{~ms}$ to retract the actuator $5 \mathrm{~cm}$, and a $75 \mathrm{~ms}$ wait. Wait times are used by the data acquisition system for computation and data transfer to the main control console.

Position of the wire is sensed by a conductive plastic film linear potentiometer connected to the end of the pneumatic actuator rod opposite the wires. Potentiometer lifetime is rated at a minimum of $2 \times 10^{7}$ cycles. Potentiometer linearity is better than .2\% over its $5 \mathrm{~cm}$ rance.

\section{Electronics and Data Acquisition System}

A block diagram of the electronics and computer data acquisition system for 20 wire scanners is shown in figure 3. Two such systems will be used for the RTM. Each set of 20 wire scanners is under the control of a secondary microcomputer station within the RTM control system 1 This secondary computer consists of a Multibus (IEEE 796) crate, two 8085 Central Processing
Unit (CPU) boards, 16K bytes of magnetic core memory, a 32-channel, 12-bit Analog to Digital Converter (ADC) board, interface boards, a CRT terminal, and a local control console. Each CPU has a Programmable Read Only Memory (PROM) for programs and $6 \mathrm{~K}$ bytes of Random Access Memory (RAM). The first CPU provides communi$c$ ation between the core memory data base and both the local and remote operator consoles. The second CPU controls the actual data acquisition and control of the wire scanners.

The linear potentiometer provides a wire position signal which is processed by a buffer amplifier and sent to a 32-channel, 12-bit ADC board. The current signal from the wire is sent to one of two amplifiers, depending on whether the electron beam is CW or pulsed. In the case of a CW beam, the current signal is sent to a high gain, DC-coupled amplifier and then to a 200 $\mathrm{MHz}$, (Wilkinson type) 13-bit, Nuclear Instrumentation Module (NIM) ADC that is interfaced to the 22-bit parallel port of the second CPU board. For pulsed beams, the wire current output goes to a charge-sensitive preamplifier and then to a NIM spectroscopy amplifier for shaping before being input to the NIM ADC. The ADC used was selected because it has the ability to convert either a varying DC level ( $C W$ case) by use of an external trigger provided by the CPU board, or a pulse peak.

Figure 4 is a block diagram of the control, data acquisition and program logic system. In operation a wirescanner is selected manually. The computer controls operation of the actuator and the choice of either $D C$ or charge-sensitive preamplifier. The gain of the $D C$ preamplifier is also computer controlled. Either preamplifier output is passed to the $A D C$ and then to the data acquisition and display system. The position of the wire is read as a voltage developed across the linear potentiometer. This signal is buffered and passed to the ADC.

\section{Performance}

Two wire scanners have been constructed and tested with $100 \mathrm{keV}$ electrons obtained from the NBS RTM chopper-buncher beam line and with $2.5 \mathrm{MeV}$ electrons from an electron Van de Graaff. Figure 5 shows the position ( $x$ axis) vs current ( $y$ axis) read out of a $1.95 \mathrm{ma} D C$ $100 \mathrm{keV}$ electron beam $2 \mathrm{~mm}$ in diameter. The photograph shows the two peaks corresponding to the two wires.

The computer data acquisition system has sampled test data at a rate of one reading every $124 \mu \mathrm{s}$ with a CPU clock rate of $2 \mathrm{MHz}$. The CPUs will run at $5 \mathrm{MHz}$ in the final configuration for a sampling time of $55 \mu \mathrm{s}$ sec.

\section{References}

1. E.R. Martin, C.M. Schneider, V.A. Martinez, R.E. Trout, and R.E. Gritzo, Proceedings of the 1981 Linear Accelerator Conference, Santa Fe, NM, P. 171.

2. M.A. Wilson, R.I. Cutler, E.R. Lindstrom, S. Penner, N.R. Yoder, D.L. Mohr, L.M. Young, and E.R. Martin, NBS-LANL Injector Installation, paper K9, this conference. 


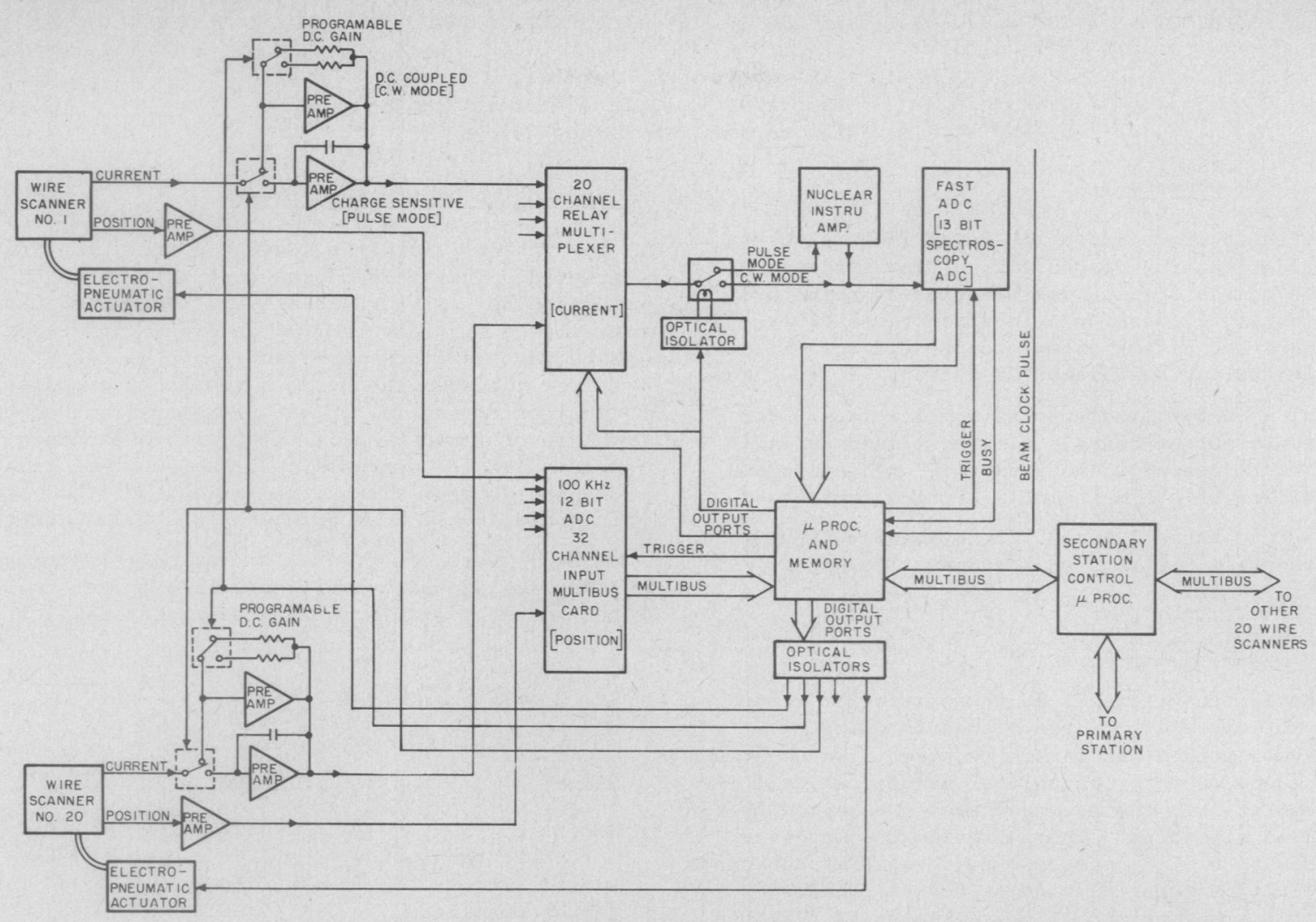

Figure 3. Wire scanner control and data acquisition electronics
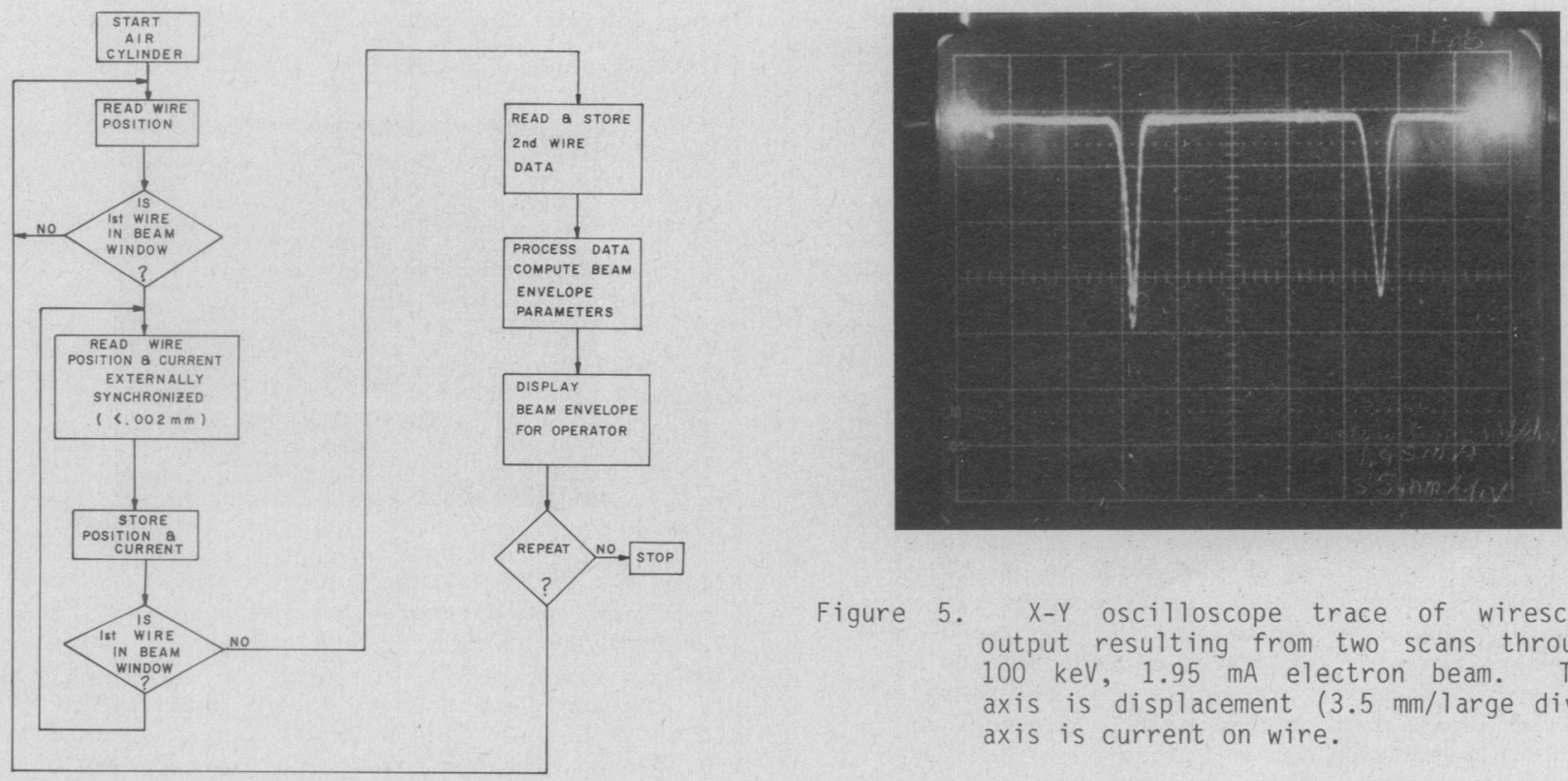

Figure 5. X-Y oscilloscope trace of wirescanner output resulting from two scans through a $100 \mathrm{keV}, 1.95 \mathrm{~mA}$ electron beam. The $X$ axis is displacement ( $3.5 \mathrm{~mm} /$ large div), Y axis is current on wire.

Figure 4. Wire scanner control and data acquisition logic diagram. 